\title{
ESCLEROSE LATERAL AMIOTRÓFICA: FISIOPATOLOGIA E CUIDADOS DE ENFERMAGEM
}

\section{LATERAL AMIOTHROPHIC SCLEROSIS: PHYSIOPATHOLOGY AND NURSING CARE}

\section{Carlos Maykis Silva Santos}

Enfermeiro. Especialista em Urgência e Emergência pela Universidade Presidente Antônio

Carlos UNIPAC-MG carlos.maykis@ hotmail.com

Daniel de Azevedo Teixeira Farmacêutico-Bioquímico Doutor em Biocombustíveis-UFVJM. Professor-adjunto da UNIPAC Teófilo Otoni - MG -danielteixeira@unipacto.com.br

Martha Honorato da Silva Enfermeira.Formada pela Universidade Unificada DOCTUM de Teófilo Otoni - MG Especialista em Urgência e Emergência e saúde da família. Professora-adjunta da universidade Presidente Antônio Carlos UNIPAC Teófilo Otoni - MG. marthahonorato@gmail.com

\section{Resumo}

A esclerose lateral amiotrófica é caracterizada como afeç̧ão neurodegenerativa e debilitante do sistema nervoso central e periférico de apresentação morfológica mais comum dentre as patologias que acometem o neurônio motor. Com incidência rara, porém elevado índice de letalidade e manifestação indiscriminada estendida à população global, a degenerescência procedente da fisiopatologia da esclerose lateral amiotrófica demonstra significativa relevância às perscrutações da ciência de enfermagem como provisão de cuidado e paliação através da sistematização da assistência de enfermagem sobrelevada nas ações e intervenções de enfermagem. O presente estudo trata-se de uma pesquisa de revisão de literatura, composta por artigos de periódicos e revistas científicas, além de livros estritos e condizentes à temática proposta. Objetivou-se mediante fundamentação científica, explicitar a fisiopatologia como aspecto científico imprescindível, relacionando-o a metodologia devidamente exposta no percurso da supracitada pesquisa, da prática do cuidado assistencial sistematizado na intervenção de enfermagem.

Palavras-chave: Esclerose lateral amiotrófica. Sistema nervoso. Neurônio motor. Cuidados de enfermagem. Assistência de enfermagem. 


\begin{abstract}
Amyotrophic lateral sclerosis is characterized as a neurodegenerative and debilitating condition of the central and peripheral nervous system with the most common morphological presentation among the pathologies that affect the motor neuron. With a rare incidence, but a high rate of lethality and indiscriminate manifestation extended to the global population, the degeneration arising from the pathophysiology of amyotrophic lateral sclerosis demonstrates significant relevance to the insights of nursing science as provision of care and palliation through the systematization of superlative nursing care in nursing actions and interventions. The present study is a literature review research, composed of articles from journals and scientific magazines, in addition to strict books and consistent with the proposed theme. The objective was based on scientific reasoning, to explain the pathophysiology as an essential scientific aspect, relating it to the methodology properly exposed in the course of the aforementioned research, of the systematic assistance care practice in nursing intervention.
\end{abstract}

Keywords: Amyotrophic lateral sclerosis. Nervous system. Motor neuron. Nursing care. Nursing care.

\title{
1 INTRODUÇÃO
}

A Esclerose Lateral Amiotrófica (ELA) designada como enfermidade neuromotora, acomete o sistema nervoso central e periférico, de etiologia hermética e progressivamente incapacitante conforme o avanço fisiopatológico, distingue-se pela degeneração gradual e irreversível dos neurônios motores, superiores e inferiores nas regiões do tronco cerebral, medula cervical, torácica e lombossacra. A ELA é a forma mais recorrente das moléstias do neurônio motor, descrita como um distúrbio generalizado de interação neuronal e subsequente associação muscular (MITCHELL; BORASIO, 2007; WIJESEKERA; LEIGH, 2009; ROWLAND; NEIL, 2001).

Genericamente, a sintomatologia clínica apresentada no desenvolvimento da ELA constitui manifestações tais como: miastenia, disfagia, fasciculações e outras provenientes de degenerações propriamente neuromusculares e/ou motoras. Todas associadas ao comprometimento funcional dos nervos cranianos, estruturas indispensáveis à sensitividade e motricidade e que se conectam ao encéfalo para a continuidade dos impulsos neuronais em condição fisiológica (STEINER, 2008).

Ainda que sua forma mais incidente seja na junção atuante em ambos neurônio motor inferior (NMI) e neurônio motor superior (NMS), existem outras variantes, tal qual a predominante no NMI, designada como atrofia muscular progressiva (AMP) e a específica do NMS, denominada de esclerose lateral primária (ELP) (COLLETTE; GUY, 2002; ROBERT; BROWN, 2013). 
O cuidado ao paciente portador da ELA distingue-se pelo caráter contínuo e paliativo, visando ratificar a fundamentação teórica do teor holístico difundido pelas ciências de saúde e minimizar a onerosidade resultante da perda na autonomia que potencializa a possibilidade de debilitação emocional e psicológica, embasada pela sistematização da assistência de enfermagem (SAE) e evidenciada na impossibilidade de realização das atividades da vida diária (AVD).

Embora a ocorrência em incidência e prevalência mundial seja rara, a letalidade e taxa de sobrevida dos indivíduos portadores dessa patologia representa um fator preocupante à população global e mesmo ao que direta ou indiretamente lida por intermédio dos cuidados e/ou intervenções paliativas de saúde requisitadas em caráter inexorável (MITCHELL; BORASIO, 2007; ROBERT; BROWN, 2013). Sendo assim, o conhecimento sistemático dos aspectos fisiopatológicos e assistenciais demonstra significativa relevância, sobretudo ao enfermeiro, como agente científico e assistencial, promotor de saúde.

O presente estudo trata-se de uma pesquisa de revisão de literatura, composta por artigos de periódicos e revistas científicas, além de livros estritos e condizentes à temática proposta. Objetivou-se mediante fundamentação científica, explicitar a fisiopatologia como aspecto científico imprescindível, relacionando-o a metodologia - devidamente exposta no percurso da supracitada pesquisa - da prática do cuidado assistencial sistematizado na intervenção de enfermagem.

\section{ASPECTOS GERAIS}

O termo esclerose morfologicamente denota, em contexto, o "enrijecimento" de determinada estrutura, conduzindo à perda de distinção funcional e o comprometimento sistêmico dos complexos subordinados e adjacentes a tal estrutura. A expressão lateral diz respeito à topografia anatômica da atuação degenerativa, especificamente na fração lateral da medula espinhal (NASCIMENTO; RODRIGUES; NUNES, 2005; SARDELLI, 2014).

Por conseguinte, o termo amiotrófico denota uma cadeia patológica desencadeadora da morte neuronal pela interrupção de suprimento tecidual, essencial à homeostasia local e exprimida clinicamente pela miastenia (NASCIMENTO; RODRIGUES; NUNES, 2005; SARDELLI, 2014). Dessarte, a caracterização da significância dos termos, demonstra o influxo típico e generalizado da fisiopatologia da ELA, imprescindível ao entendimento exordial. 
O primeiro relato de similitude sintomatológica foi descrito em 1830 por Sir Charles Bell, cirurgião e anatomista, atentando à associação nervosa das evidências aferidas, tais como paresias contrastadas com a preservação de algumas sensibilidades. Posteriormente por meio de outros neurologistas estabelecida "atrofia muscular progressiva" como denominação própria, porém relativamente genérica (SARDELLI, 2014).

Em 1869, o complexo neuropatogênico foi preliminarmente clarificado por Jean Charcot, professor de neurologia, caracterizando a paralisia bulbar progressiva e a ELP embora distinguindo da ELA, delimitando os aspectos periféricos e estabelecendo as bases científicas fundamentais para a distinção diagnóstica e aos estudos que se seguiram (SARDELLI, 2014).

A terminologia sinonímia a ELA embora menos recorrente, "doença do neurônio motor" foi prescrita em 1933 com a finalidade de unificar as especificidades observadas anteriormente e classificá-las como subtipos (SARDELLI, 2014). Não obstante, em meados das décadas de 20 a 40, a ELA torna-se correntemente intitulada como doença de Lou Gehrig, dada amplitude de sua popularidade nos Estados Unidos da América, como sendo personalidade ínclita do âmbito dos esportes além de vítima fatal da ELA (SARDELLI, 2014).

No Brasil, o primeiro relato de elucidação científica estrita, antecedido de estudos clínicos e epidemiológicos, é datado de 1909, com a publicação de um caso clínico no revista "Brazil-Medico" pelo Dr. Cypriano de Freitas, professor da faculdade de medicina do Rio De Janeiro, insigne pela observação diligente das manifestações sintomáticas e da idiossincrasia progressiva para a precisão diagnóstica (SARDELLI, 2014; QUADROS, 2006).

Posteriormente ao estudo clínico do Dr. Cypriano, disposto evidentemente como anteparo fomentador dos estudos porvindouros, haja vista o gradativo todavia subsecutivo interesse acadêmico e agudeza científica, o Dr. Gonçalves Viana procede à investigação de dois casos clínicos, cuja defesa da própria tese foi o substrato oriundo dos estudos realizados no mesmo paciente de um dos casos clínicos supracitados conduzindo- o a considerações categóricas da fisiopatologia, dantes de percepção hermética no Brasil (SARDELLI, 2014; QUADROS, 2006).

Ainda que estudos em torno de uma causa comum ou relativamente metódica desde os primórdios sejam evidentes, a ELA circunscreve uma multiplicidade de fatores e derivações desencadeantes seguintes: excitotoxicidade (VAROQUAUX et al., 2002); autoimunidade através de estímulos extrínsecos ou condições genotípicas (NORDON; ESPÓSITO, 2009); estresse oxidativo (D’AMICO et al., 2013); lesão mitocondrial (MENZIES; INCE; SHAW, 2002); disfunções por 
polimorfismos em subunidades dos neurofilamentos (TOMKINS, 1998); apoptose (SATHASIVAM; INCE; SHAW, 2001), entre outras teorias que complexificam a possibilidade de um consenso causal.

A incidência da ELA de acordo com Worms (2001) na década de 90 situava-se 1,47 a 2,70 casos em cada 100.000 habitantes por ano, conduzindo a média de 1,89 casos em cada 100.000 habitantes por ano. Entretanto, para Oliveira e Pereira (2009), a mesma incidência varia de 0,2 a 2,5 casos em 100.000 habitantes por ano. Supletivamente, a forma esporádica, manifestante desvinculada de critérios hereditários, segundo Mitchell e Borasio (2007) possui incidência "entre 1,5 a 2 casos em cada 100.000 habitantes por ano".

Ainda referente aos dados epidemiológicos, Neto e Takayanagui (2013)

[...] a ocorrência é mais elevada nos homens do que nas mulheres, na proporção de 1,5 por 1 , a idade média para o diagnóstico varia de 40 a 70 anos, com uma esperança média de vida de 2 a 5 anos. Dez por cento dos pacientes com ELA vão viver durante pelo menos dez anos. A incidência da esclerose lateral amiotrófica, conforme reavaliada recentemente pelo grupo europeu EURALS, é de 2,16 casos por 100.000 habitantes, sendo maior no sexo masculino $(2,4 / 100.000)$ do que no sexo oposto (1,9/100.000). A idade mais frequente de início nas mulheres é de 75 a 79 anos, e, nos homens, está no intervalo de 70 a 74 anos.(NETO; TAKAYANAGUI, 2013)

No mesmo padrão, os dados sobre a prevalência se distinguem, com prevalência de cerca de 6 casos em cada 100.000 habitantes por ano segundo Mitchell e Borasio ${ }^{1}$ e de em média 5,2 casos em cada 100.000 habitantes por ano em conformidade com Worms (2001).

A taxa de mortalidade da ELA perfaz cerca de 1,54 a 2,55 em cada 100.000 habitantes por ano, resultando em média de 1,91 em cada 100.000 habitantes por ano (WORMS, 2001) e variando tenuemente entre regiões e países, como por exemplo os Estados Unidos que até 2001 possuía o equivalente a 2,13 em cada 100.000 habitantes por ano (SEJVAR et al., 2005).

\section{FISIOPATOLOGIA}

O mecanismo fisiopatológico da ELA abrange vários fatores que ora se consubstanciam às hipóteses causais, entretanto é estabelecido sobretudo como o pressuposto fundamental a degeneração 
seletiva dos neurônios motores do córtex motor primário e dos cornos anterolaterais da medula espinhal.

Difundir tal mecanismo, mesmo academicamente objetiva desvelar o liame de desconhecimento e a disfunção do lidar como consequência desta, embora a priori, seja fundamentalmente necessário para profissional de saúde como agente científico a compreensão acerca dos termos e conceitos que permeiam a neurofisiologia.

O neurônio, célula de unidade funcional e estrutural do sistema nervoso é o instrumento de difusão do impulso elétrico que preserva a homeostasia e permite a manutenção orgânica dos tecidos que compõem desde as glândulas até os órgãos em perspectiva sistemática (ROWLAND; NEIL, 2001; HAMMER; STEPHEN, 2016).

Biologicamente similar à disposição morfológica da maioria das células, possui um corpo nuclear, além do axônio, estrutura ramificante de comunicação aos neurônios adjacentes e os dendritos, pequenas porções ramificadas anexadas ao corpo nuclear que recebem os impulsos elétricos propriamente ditos (ROWLAND; NEIL, 2001; HAMMER; STEPHEN, 2016).

A extensão comunicante do axônio permite a terminação interativa em outros neurônios, denominada sinapse, frequente entre axônios e excepcionalmente entre dendritos, é processo que proporciona a efetiva transmissão do fluxo elétrico das informações e células, e se ausente resulta inevitavelmente no desequilíbrio eletrolítico generalizado (ROWLAND; NEIL, 2001; HAMMER; STEPHEN, 2016).

Situados na região do tronco encefálico, - evidentemente oriundos do córtex motor - medula espinhal e estendidos pelos axônios aos nervos espinhais estão os neurônios motores, estruturas especializadas, diferenciadas e responsáveis pela ação motora e reflexa, além do trofismo muscular e nervoso, sub-classificados em superior e inferior, conjuntamente associados à iniciação e cessação de todo movimento voluntário (ROWLAND; NEIL, 2001; HAMMER; STEPHEN, 2016).

Lesões atuantes no vasto complexo de quaisquer dos neurônios motores demonstram manifestações clínicas distintas, evidenciadas principalmente por paralisias flácidas, alterações na tonicidade motora e reflexos, alternadas entre agudos e crônicos (HAMMER; STEPHEN, 2016).

A degeneração frequentemente bilateral dos neurônios motores no córtex motor primário e cornos anterolaterais da medula espinal, nominalmente os NMS e NMI, na ELA, como salientado anteriormente, alberga diversos fatores desde bioquímicos a genéticos, portanto, semelhantemente às 
hipóteses etiológicas, não se extenuam os esforços dispendidos a fim de relimar as considerações vigentes (VENTURIN, 2012).

Um dos mecanismos de elucidação fisiopatológica, diz respeito a excitotoxicidade ao neurotransmissor glutamato, associada a discrepância na cadeia de codificação genética. A atuação do glutamato suscita a maximização da concentração do íon de Cálcio intracelular no citosol do neurônio pós-sináptico, propagando o seu devido potencial (ROWLAND; NEIL, 2001; GREENBERG; AMINOFF; SIMON; 2014).

O influxo maximizado do íon de Cálcio no ambiente intracelular, ativa enzimas estritamente coadunáveis ao cálcio que removem o glutamato da sinapse por vias de mecanismos sinalizadores da célula pós-sináptica. A subsistência dessa condição intracelular exacerbada, na ausência do mecanismo enzimático acarreta na excitotoxicidade e por conseguinte na apoptose.

Observa-se nesse contexto que a via de correspondência genética permite que a possibilidade da implícita disfuncional seja intrínseca à proteína transportadora por conseguinte justifica na incorreta inserção do seu respectivo ácido ribonucleico mensageiro (ROWLAND; NEIL, 2001).

Outra sintaxe de referência cientificamente plausível na excitotoxicidade, relaciona a ELA a mutações genéticas do citosólico cobre-zinco superóxido dismutase (SOD1), situado no cromossomo 21, que alterariam sua funcionalidade, gerando por sua vez radicais de hidroxila pela catalisação da redução dos níveis de peróxido de hidrogênio, além de outras mutações que elevariam o teor de SOD1 possibilitando a neurotoxicidade. As alegações argumentativas sobre o estresse oxidativo elucubram o potencial nocente de variantes moleculares de oxigênio, provindas das referidas mutações do SOD1(ROWLAND; NEIL, 2001; CAVACO, 2016).

De outro modo, as evidências polimórficas nas subunidades dos filamentos dos neurônios, suportam a tese da influência cito esquelética. Provenientes de inclusões mutacionais, a insuficiência no transporte de moléculas e a desestruturação do axônio como efeitos dessas anomalias morfológicas, fatalmente conduziriam a morte dos neurônios motores (ROWLAND; NEIL, 2001).

A apoptose, igualmente, demonstra significante relevância no transcorrer fisiopatológico da ELA. Baseados nos achados clínicos post-mortem notam-se condensados nucleares e citoplasmáticos adjuntos a corpos apoptóticos, embora não sejam ratificadas categoricamente como constatações irrefragáveis, pela necessidade da análise da fragmentação do ácido desoxirribonucleico dos neurônios 
motores minuciosamente e em uma incidência de proporção 1 para cada 100 (ROWLAND; NEIL, 2001).

\section{DIAGNÓSTICO E TRATAMENTO}

As manifestações sintomatológicas da ELA geralmente apresentam frequência súbita e caráter indistinguível, precipitando ao diagnóstico considerável detença que atinge segundo Neto e Takayanagui (2013) "em média em 12 meses." Entre o transcorrer do início dos primeiros sintomas e o estabelecimento inequívoco do diagnóstico, o enfermo é submetido a diversos procedimentos cintilográficos e exames complementares (NETO; TAKAYANAGUI, 2013).

O diagnóstico da ELA ratifica cientificamente um conjunto de sinais proeminentes e objetivos, de comprometimentos do NMI através de exame clínico, eletroneuromiográfico ou neuropatológico imiscuído ao comprometimento do NMS, sob extensão crônica e degeneração progressiva (NETO; TAKAYANAGUI, 2013). Apresentações tipificadas como astenia, paralisia e sinal de Babinski, configuram ubiquamente a nominata de evidências (CAVACO, 2016).

A propedêutica neurológica assim sendo, representa aspecto substancial para o estabelecimento do diferencial diagnóstico e dispõe de critérios como, aspecto muscular, que atenta quanto às evidências táteis de hipotrofia e atrofia muscular, fasciculações e espasmos reflexos ou extensores; tônus muscular, condizente a contratura, espasticidade, hipotonia e paratonia; força muscular, permitindo a observação mensurada, através da graduação das respostas; coordenação motora; percussão reflexa superficial e dos tendões musculares; avaliação da marcha quanto à distribuição dos membros e postura deambulatória, entre outros variados instrumentos de investigação (GREENBERG; AMINOFF; SIMON; 2014).

Apesar da inespecificidade do amplo quantitativo de sintomas neurológicos presumíveis ao dano motor, a significância dos dados colhidos permite a explanação do diagnóstico diferencial, ferramenta imperativa quando na sujeição do paciente aos exames, possibilitando reconhecimento precoce da degeneração atuante, com vias à provisão na ciência patológica e prática assistencial seja na adaptação do ciclo doméstico ou até em cuidados paliativos. 
A despeito da ausência de tratamento de ação retroativa a neurodegeneração perpetuante, o fármaco riluzol permitido pela Food and Drug Administration (FDA), de ação antiglutametérgico demonstra maior expressividade e eficácia atuante na maximização da taxa de sobrevida. Embora não totalmente elucidado, infere-se que o mecanismo de ação do riluzol compreenda na mediação responsiva do receptor agonista do glutamato, N-metil-D-aspartato (NMDA), suprimindo a difusão do referido neurotransmissor dos sítios sinápticos pela manutenção da inatividade dos canais de sódio na conjunção da resposta de captação glutâmica extracelular (DISTAD et al., 2008; LYALL et al., 2001).

Em decorrência de tais fatores, e contrariando expectativas incipientes em torno da eficácia medicamentosa como via resolutiva, torna-se explícito o caráter ainda insolúvel portanto paliativo do referido fármaco ante a inexorabilidade da degeneração, que entretanto viabiliza a oportunização de extensão da sobrevida anteriormente abordada.

A insuficiência respiratória, complicação mais recorrente no portador de ELA, propicia a intervenção por um enfático procedimento de tratamento não farmacológico da ELA, que consiste na terapia de ventilação mecânica não invasiva (VMI), visando minimizar as consequências face à analose da musculatura respiratória. Sob prescrição médica, a VMI objetiva ofertar suporte respiratório através da pressão positiva proporcionada (DISTAD et al., 2008; MILLER et al., 2009).

Em perspectiva de denotação invasiva, a traqueostomia figura como alternativa respiratória, certa feita que os agravos da progressão impreterível suscitem maior frequência em assistência ventilatória. Outra intervenção diz respeito a monitoração e planejamento da condição nutricional na contrapartida das disfunções alimentares coadjuvantes como perda de massa corpórea, disfagia e broncoaspiração (KODAMA; SPURAS; PADULA, 2009).

Em suma, as formas de tratamento supracitadas enfatizam a possibilidade da ampliação da taxa de sobrevida, desconsiderando a possibilidade de ação motora responsiva pelo caráter terminal e degenerativo. Para os profissionais de saúde, mormente o assistencial, estar ciente das idiossincrasias que circundam o tratamento, é, por conseguinte requisito de eficácia do embasamento científico.

\section{ASSISTÊNCIA DE ENFERMAGEM}


O enfermeiro lida profissionalmente com vastas complexidades práticas na sistematização de enfermagem, gerenciando cuidados e avaliando criteriamente as intervenções em acordo com as necessidades identificadas, desde procedimentos relativamente banais até cuidados contínuos e capacitações em saúde com fim de respaldar a prática e aperfeiçoar o cuidado holístico.

O transcurso do adoecer costuma conglomerar múltiplos fatores que carecem da assistência, criticidade e objetividade. Alterações ressalientes e súbitas no padrão de vida do portador de ELA necessitam de instruída adaptação às condições estabelecidas assim como quaisquer enfermidades graves e incapacitantes, avultando assim a relevância da intervenção idônea do profissional assistencial de saúde, inerentemente enfermeiro.

Dentre as complicações potenciais abrangidas pela intervenção de enfermagem, a úlcera por pressão figura com alta relevância, vez que o paciente acometido pela atrofia muscular condizente a fisiopatologia da ELA, conserva a imobilização corporal por período prolongados, dada a completa derrogação de sua autossuficiência. A úlcera por pressão é oriunda da redução do aporte sanguíneo e da oxigenação, em determinado sítio por lesão gradativa (NASCIMENTO; RODRIGUES; NUNES, 2005; CAVALCANTE E. S.; CAVALCANTE C. A. A.; FRANCISCO, 2013).

A intervenção de enfermagem portanto, para esse óbice, compreende estrategicamente a ação profilática de mudança de decúbito em período avaliado pelo enfermeiro, visando suavizar a pressão atuante no ponto de contato entre as proeminências ósseas e a pele e descomprimir a tensão de forças de cisalhamento principalmente durante a mobilização do paciente (NASCIMENTO; RODRIGUES; NUNES, 2005; CAVALCANTE E. S.; CAVALCANTE C. A. A.; FRANCISCO, 2013).

Outra intercorrência possível é a baixa autoestima experimentada pelo déficit de autocuidado, sendo assim, além dos familiares e cuidadores o paciente portador de ELA deve ser informado e esclarecido acerca de quaisquer pontos que possivelmente apresentem ambiguidade, ratificando a conduta humanística no tratamento quando o paciente cônscio dos procedimentos consente com seu auxiliador.

A manutenção do cuidado promove a possibilidade excepcional da preservação de alguma faculdade motora, ainda que rudimentarmente, como na capacidade de deglutir. Medidas compensatórias integrado no cuidado são designadas para minimizar as debilidades provenientes das disfunções inerentes a ELA, como movimentação e adaptação avaliadas evidentemente pela equipe multidisciplinar para resposta multifatorial (NASCIMENTO; RODRIGUES; NUNES, 2005). 
A linguagem de sinais, mímica, e a expressão através de sistemas eletrônicos computacionais podem ser utilizados como alternativas eficazes na comunicação verbal e não verbal, sendo ferramentas egrégias para refrear o isolamento social.

Por intermédio da SAE o enfermeiro desempenha ações de estímulo e desenvolve o planejamento de cuidados, ponderando a viabilidade e mensurando a efetividade de cada intervenção proposta, evitando desse modo que as complicações pósteras ao longo do tratamento sejam mitigadas, desenvolvendo bilateralmente o papel essencial educador e interventor de saúde.

Alguns exemplos de diagnósticos de enfermagem respaldados pela NANDA (North American Nursing Diagnosis Association) e que podem abranger as condições clínicas do portador de ELA são: Comunicação prejudicada associada a alterações do sistema nervoso e evidenciada por utilização de expressão gestual; mobilidade prejudicada associada ao distúrbio neuromuscular; nutrição desequilibrada menor que as necessidades corporais, relacionada à incapacidade de ingerir alimentos e evidenciada por disfagia; interação social prejudicada associada função social prejudicada e evidenciada pela disfunção de comunicação; eliminação urinária prejudicada associada à dano na sensibilidade motora, evidenciado por frequência e urgência vesical; ansiedade associada ao risco ou alteração das condições de saúde do ambiente, da relação familiar e interpessoal e padrão financeiro, evidenciada por infelicidade em decorrência das mudanças nas condições de vida; padrão respiratório ineficaz associado à disfunção neuromuscular evidenciado por dispneia, risco de lesão associada à mobilidade restringida e perda da integridade da pele; risco de Infecção associado à procedimentos invasivos; (GARCEZ, 2015).

Subsequentemente aos diagnósticos de enfermagem e mantendo a correspondência entre o conhecimento científico e identificação das anormalidades concretas e potencias, se estabelece intervenções de enfermagem, não exaustivas, dentre outras que correspondem na utilização colchão piramidal, mensuração do nível de consciência por intermédio escala de coma de Glasgow, do risco de desenvolvimento de úlcera por pressão pela escala de Braden e do risco de queda pela escala de Morse (JOHNSON et al., 2012).

Assepsia das mãos precedendo a quaisquer procedimentos assistenciais, avaliação dos sinais vitais mediante registro e comparação dos dados obtidos e da integridade da pele nos locais de punção de acessos venosos periféricos e centrais atentando para sinais flogísticos e alterações exógenas, preservar a higiene corporal com periodicidade ponderada, elevar a cabeceira no leito quando em conformidade com orientação médica, atentar quanto o posicionamento do tubo orotraqueal (TOT) e 
pressão do balonete, bem como o aspecto e coloração da secreção traqueal aspirada pelo TOT, datação e troca de equipo na consumação de infusão ou anteriormente quando constatadas intercorrências tópicas ou funcionais, controle de posicionamento e fixação das sondas nasais e vesicais, registro do balanço hídrico, dentre outras intervenções que permitem subjulgar intercorrências contingentes (JOHNSON et al., 2012).

O enfermeiro dispõe ainda da possibilidade da capacitação da família, - necessitante de um parecer objetivo sob a ótica do profissional de íntima - na aptidão nos cuidados não complexos e na provisão emergencial, esclarecendo contingências sob fundamentação fisiopatológica em hipóteses e anseios que emergem ante a hermeticidade e conteúdo insólito, disposto em ELA.

\section{CONSIDERAÇÕES FINAIS}

A interação entre a ciência e a prática de enfermagem revela notável distinguibilidade, posto que o espectro do conhecimento na avaliação para intervenção de enfermagem se porta cadente certa feita que a profissão de enfermagem é difundida como arte do cuidar em detrimento do cunho científico embasador.

Compreender a fisiopatologia consiste na problematização e posterior simplificação dos mecanismos que regem a progressão de uma determinada patologia desde sua contração até inevitável involução ou evolução. Associar portanto os conhecimentos científicos auferidos pela fisiopatologia viabilizam a criterização do cuidado e o estabelecimento objetivo das intervenções em saúde.

Como explanado neste estudo, a esclerose lateral amiotrófica constitui-se de uma enfermidade neurológica grave e fatal, apresentando demasiada relevância a assistência de enfermagem pelo caráter progressivamente debilitante que conduz inevitavelmente aos cuidados contínuos e criteriosos requisitantes do raciocínio clínico.

A intervenção de enfermagem tem por objetivo consumar as constatações obtidas na avaliação de enfermagem e posterior diagnóstico. Em suma, a fisiopatologia da esclerose lateral amiotrófica associada a intervenção, propicia em evidente conforme disposto, o aprimoramento da minimização da 
proveniências debilitantes não inerentes ao progredir sublimado no rigor holístico das ciências de enfermagem.

\title{
AMIOTROPHIC LATERAL SCLEROSIS: PHYSIOPATHOLOGY AND NURSING CARE
}

\author{
Carlos Maykis Silva Santos, Daniel de Azevedo Teixeira **
}

\begin{abstract}
Amyotrophic lateral sclerosis is characterized as a neurodegenerative and debilitating condition of the central and peripheral nervous system of morphological presentation more common among the pathologies that affect the motor neuron. With a rare incidence, but a high index of lethality and indiscriminate manifestation extended to the global population, degeneration from the pathophysiology of amyotrophic lateral sclerosis demonstrates a significant relevance to nursing science as a provision of care and palliation through the systematization of nursing care elevated in the actions and interventions. The present study is a review of the literature, composed of articles from periodicals and scientific journals, as well as books that are strict and consistent with the proposed theme. The objective of this study was to explain the pathophysiology as an essential scientific aspect, and to relate it to the methodology duly exposed in the aforementioned research, of the practice of assisted care systematized in the nursing intervention.
\end{abstract}

Keywords: Amyotrophic lateral sclerosis. Nervous system. Motor neuron. Nursing care. Nursing care.

\section{REFERÊNCIAS}


CAVACO, S. G. Esclerose lateral amiotrófica: fisiopatologia e novas abordagens farmacológicas. Faculdade de Ciências e Tecnologia da Universidade do Algarve. Algarve. 2016.

CAVAlCANTE, E. S.; CAVAlCANTE, C. A. A.; FRANCISCO, A. N. M. Assistência de enfermagem ao paciente com traumatismo raquimedular. ReseachGate, 2013. Disponivel em: $<$ https://www.researchgate.net/publication/259005818_ASSISTENCIA_DE_ENFERMAGE M_AO_PACIENTE_COM_TRAUMATISMO_RAQUIMEDULAR?enrichId=rgreq587a9f1a39f05b664d01c67780809171 XXX\&enrichSource=Y292ZXJQYWdlOzI1OTAwNTgxODtBUzo5ODg5Mzk0NTYzODkx MkAxNDAwNTg5NTYyMTAw\&el=>. Acesso em: 10 Mai. 2018.

CHIEIA, M. A. T.; OLIVEIRA, A. S. B. Esclerose lateral amiotrófica. In: NETO, J. P. B.; TAKAYANAGU, O. M. ratado de neurologia da academia brasileira de neurologia. $1^{\text {a }}$. ed. Rio de Janeiro: Elsevier, 2013. Cap. 73.

COLlETTE, K. H.; GUY, A. R. Familial amyotrophic lateral sclerosis. Wiley Online Library, 2002. Disponivel em: <https://onlinelibrary.wiley.com/doi/abs/10.1002/mus.10001>. Acesso em: 2 Mar. 2018.

D'AMICO, E. et al. Clinical perspective on oxidative stress in sporadic amyotrophic lateral sclerosis. ScienceDirect, 2013. Disponivel em: <https://www.sciencedirect.com/science/article/pii/S0891584913003109?via\%3Dihub>. Acesso em: 21 Mar. 2018.

DISTAD, B. J. et al. Drug therapy in amyotrophic lateral sclerosis. ScienceDirect, 2008. Disponivel em: <https://www.sciencedirect.com/science/article/pii/S1047965108000338?via\%3Dihub>. Acesso em: 26 Abr. 2018.

GARCEZ, R. M. Diagnósticos de Enfermagem da Nanda: Definições e Classificação. 10a . ed. Porto Alegre: ARTMED, 2015.

GREENBERG, D. A.; AMINOFF, M. J.; SIMON, R. P. Distúrbios motores. In: GREENBERG, D. A.; AMINOFF, M. J.; SIMON, R. P. Neurologia clínica. 8a . ed. Porto Alegre: AMGH, 2014. Cap. 9, p. 234-303. 
HOERTH, C. L. Distúrbios do Sistema Nervoso. In: HAMMER, G. D.; STEPHEN, J. M. Fisiopatologia da doença: uma introdução à medicina Clínica. $7^{\mathrm{a}}$. ed. Porto Alegre: AMGH, 2016. Cap. 7, p. 145-186.

JOHNSON, M. et al. Ligações NANDA NOC - NIC Condições Clínicas: Suporte ao Raciocínio e Assistência de Qualidade. 3ª . ed. Rio de Janeiro: Elsevier, 2012.

KODAMA, C. M.; SPURAS, M. V.; PADULA, M. P. C. Cuidados prestados pelos enfermeiros aos pacientes de reabilitação. Arq Med Hosp Fac Cienc Med Santa Casa São Paulo, 2009. Disponivel em: <http://www.fcmsantacasasp.edu.br/images/Arquivos_medicos/2009/54_3/vlm54n3_4.pdf>. Acesso em: 7 Maio 2018.

LYALL, R. A. et al. A prospective study of quality of life in ALS patients treated with noninvasive ventilation. Neurology, 2001. Disponivel em: <http://n.neurology.org/content/57/1/153.long>. Acesso em: 26 Abr. 2018.

MENZIES, F. M.; INCE, P. G.; SHAW, P. J. Mitochondrial involvement in amyotrophic lateral sclerosis. Europe PMC, 2002. Disponivel em: <http://europepmc.org/abstract/med/11850111>. Acesso em: 21 Mar. 2018.

MILLER, R. G. et al. Practice parameter update: the care of the patient with amyotrophic lateral sclerosis: drug, nutritional, and respiratory therapies (an evidence-based review): report of the quality standards subcommittee of the american Academy of Neurology. Neurology, 2009. Disponivel em: <http://n.neurology.org/content/73/15/1218.long>. Acesso em: 2 Maio 2018.

MITCHELL, J. D.; BORASIO, G. D. Amyotrophic lateral sclerosis. The Lancet, 2007. Disponivel em: <https://www.thelancet.com/journals/lancet/article/PIIS0140-6736(07)609441/fulltext>. Acesso em: 2 Mar. 2018.

NASCIMENTO, J. W.; RODRIGUES, L. C.; NUNES, R. C. O. M. Atuação do enfermeiro frente a pacientes com esclerose lateral amiotrófica. Simpósio de TCC e Seminário de IC, 2005 . c4664123d653dd99899adb08da5e84a.pdf>. Acesso em: 5 Mar. 2018. 
NORDON, D. G.; ESPÓSITO, S. B. Atualização em esclerose lateral amiotrófica. Faculdade de Ciências Médicas de Sorocaba, v. XI, n. 2, p. 1-3, 2009.

OLIVEIRA, A. S. B.; PEREIRA, R. D. Amyotrophic lateral sclerosis (ALS): three letters thatchange the people's life. Scielo, 2009. Disponivel em: $<$ http://www.scielo.br/scielo.php?pid=S0004282X2009000400040\&script=sci_abstract\&tlng $=\mathrm{pt}>$. Acesso em: 12 Abr. 2018.

OLIVEIRA, M. A. T. C. A. S. B. Esclerose lateral amiotrófica. In: NETO, J. P. B.; TAKAYANAGUI, O. M. Tratado de neurologia da academia brasileira de neurologia. $1^{\text {a }}$. ed. Rio de Janeiro: Elsevier, 2013. Cap. 73.

PEREIRA, R. D. B. Epidemiologia: ELA no Mundo. Neurociências, v. 14, p. 9-13, abr/jun 2006.

QUADROS, A. A. J. História da esclerose lateral amiotrófica no Brasil. Neurociências, v. XIV, p. 14-23, abr/jun 2006.

ROBERT, H.; BROWN, J. R. Amyotrophic lateral sclerosis and other motor neuron diseases. In: STEPHEN, H. Harrison's Neurology in Clinical Medicine. $3^{\text {a }}$. ed. [S.1.]: McGraw-Hill Education, 2013. Cap. 32, p. 370-379.

ROWLAND, L. P.; SHNEIDER, N. E. A. Amyotrophic lateral sclerosis. The New England Journal of 2001.2 Disponivel em: $<$ https://www.researchgate.net/publication/11953427_Amyotrophic_Lateral_Sclerosis>. Acesso em: 2 Mar. 2018.

SARDELLI, C. Esclerose lateral amiotrófica (ELA), 2014. Disponivel em: <https://www.al.sp.gov.br/propositura/?id=1200199>. Acesso em: 5 Mar. 2018.

SATHASIVAM, S.; INCE, P. G.; SHAW, P. J. Apoptosis in amyotrophic lateral sclerosis: a review of the evidence. Wiley Online Library, 2001. Disponivel em: <https://onlinelibrary.wiley.com/doi/pdf/10.1046/j.0305-1846.2001.00332.x>. Acesso em: 2 Abr. 2018.

SEJVAR, J. et al. Amyotrophic lateral sclerosis mortality in the United States, 1979-2001. Karger, 2005. Disponivel em: <https://www.karger.com/Article/Pdf/86679>. Acesso em: 21 Abr. 2018. 
STEINER, A. N. F. Profissionais de saúde na relação com os pacientes portadores de esclerose lateral amiotrófica: aspectos psicológicos e de qualidade de vida. Universidade de São Paulo. São Paulo. 2008.

TOMKINS, J. et al. Novel insertion in the KSP region of the neurofilament heavy gene in amyotrophic lateral sclerosis (ALS). Ovid, 1998. Disponivel em: <https://insights.ovid.com/pubmed?pmid=9875737>. Acesso em: 2 Abr. 2018.

VAROQUAUX, O. S. et al. Glutamate levels in cerebrospinal fluid in amyotrophic lateral sclerosis: a reappraisal using a new HPLC method with coulometric detection in a large cohort of patients. Journal of the Neurological Sciences, 2002. Disponivel em: <https://www.elsevier.com/locate/jns>. Acesso em: 15 Mar. 2018.

VENTURIN, G. T. Potencial terapêutico das células mononucleares da medula óssea em um modelo experimental de esclerose lateral amiotrófica. Pontifícia Universidade Católica do Rio Grande do Sul. Porto Alegre. 2012.

WIJESEKERA, L. C.; LEIGH, P. N. Amyotrophic lateral sclerosis. Orphanet Journal of Rare Diseases, 2009. Disponivel em: <https://ojrd.biomedcentral.com/articles/10.1186/17501172-4-3>. Acesso em: 2 Mar. 2018.

WORMS, P. M. The epidemiology of motor neuron diseases: a review of recent studies. ScienceDirect, 2001. Disponivel em: <https://www.sciencedirect.com/journal/journal-of-theneurological-sciences/vol/191/issue>. Acesso em: 12 Abr. 2018. 